\title{
Conductor Position Optimization of a Transmission Line Excitation Chamber
}

\author{
Mario Alves Santos Jr. ${ }^{1,2}$, Sergio L. Avila ${ }^{1}$, Carlos Antonio Franca Sartori ${ }^{1}$, Djonny Weinzierl ${ }^{1,3}$, \\ Laurent Krähenbühl $^{4}$, Luiz Lebensztajn ${ }^{1}$, and Jose Roberto Cardoso ${ }^{1}$ \\ ${ }^{1}$ Laboratório de Eletromagnetismo Aplicado LMAG/PEA/EPUSP, 05508-900 São Paulo/SP, Brazil \\ ${ }^{2}$ Centro Tecnológico da Marinha em São Paulo, 05508-000 São Paulo/SP, Brazil \\ ${ }^{3}$ Centro Universitário Jaraguá do Sul, 89254-430 Jaraguá do Sul/SC, Brazil \\ ${ }^{4}$ Ampère (CNRS UMR5005), Université de Lyon, Ecole Centrale de Lyon, 69134 Ecully Cedex, France
}

\begin{abstract}
This paper presents a hybrid mono/multiobjective optimization approach based on numerical, statistical, and fitness evaluation concerning the conductor positions in a transmission line excitation chamber (TLEC). The field profile and the related indexes of merit, as well as statistical ones, regarding the working volumes (WVs) are used to evaluate the chamber configuration performance. The compromise between WV and $E$-field, constrained by the $E$-field standard deviation, is shown through Pareto's front. An index of merit based on area calculation under Pareto's front is defined and used for transmission line set comparison.
\end{abstract}

Index Terms-Electromagnetic compatibility, optimization methods.

\section{INTRODUCTION}

I N previous works, the transmission line excitation chamber (TLEC) performance evaluations have been carried out using different approaches. These were presented in [1] and [2] in which finite-integration technique (FIT), transmission line method (TLM), and also an optimization tool were used to evaluate the field uniformity within the working volume (WV) on a predefined TLEC configuration. The TLEC configuration here presented is based on three-conductor phase-shifting excitation configuration and proposed as an alternative when low frequencies are taken into consideration [3]. Fig. 1 shows a sketch of a three-wire reverberation chamber configuration. Thus, the optimization problem aims to maximize the WV and the $E$-field, which is restricted to uniformity constraints. The $\mathrm{WV}$ is the locus where the object under test will be placed.

In this work, the main focus is to find the better transmission line configurations, i.e., wire positions, which present the best TLEC performance related to the aforementioned indexes. A good TLEC performance is associated to a high WV and $E$-fields average values. To obtain the wire configuration and the corresponding TLEC performance, a numerical approach has been implemented by applying multiobjective optimization. Deterministic and statistical approaches are applied in the analysis.

\section{HYBRID OPTIMIZATION PROCEDURE}

A WV is assumed to be a region inside the reverberation chamber (Fig. 1) where the homogeneity of the electric field attains a prescribed level. EMC tests could be performed only with this minimum homogeneity level, and the mean value within the WV should be as high as possible considering low input power.

Manuscript received December 23, 2009; revised February 25, 2010; accepted March 02, 2010. Current version published July 21, 2010. Corresponding author: M. A. Santos Jr. (e-mail: mario_alves@pea.usp.br).

Color versions of one or more of the figures in this paper are available online at http://ieeexplore.ieee.org.

Digital Object Identifier 10.1109/TMAG.2010.2045360

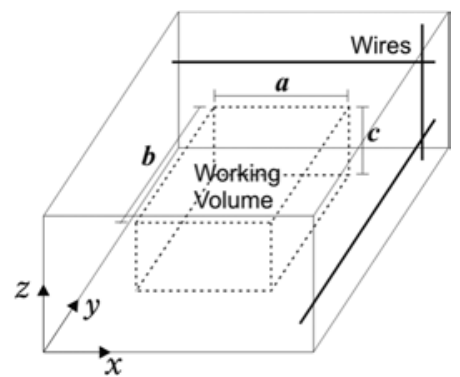

Fig. 1. Sketch of the three-wire reverberation chamber configuration.

In this work, a high-performance TLEC must be as flexible as possible, i.e., it can be used for EMC tests for small or big devices. For a given input power, when a small device is tested inside the TLEC, high $E$-fields can be obtained. Nevertheless, when big devices are tested, only low fields are achieved. So we have two kinds of problems:

1) obtain an index to measure the flexibility of the chamber for a given TLEC, characterized by the positions of the wires;

2) with this index, use a reliable algorithm to compare different TLEC configurations, in order to obtain the best configuration.

In this work, we will adopt an index entitled LA to measure the flexibility of the TLEC, which will be well described in the next section. Fig. 2 shows a sketch of the adopted procedure. It consists of three blocks: the electric field computation, which provides the characterization of the chamber; the characterization block, which consists of a multiobjective problem that analyzes the contradiction between the $\mathrm{WV}$ and the electric field in order to calculate LA; and the optimization problem, in order to maximize LA.

\section{A. Coupling the Field Computation Software and the Optimization Environment}

The adopted procedure has five fundamental steps:

1) random positions for TL are generated;

2) TL positions are charged to the field computation software that uses FIT [5]; 


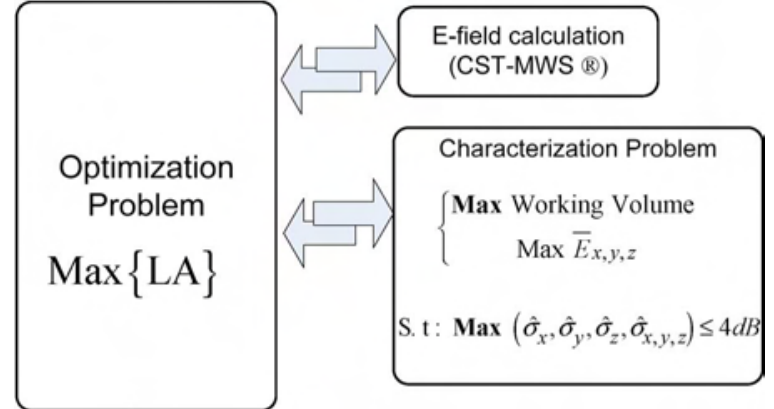

Fig. 2. Hybrid optimization procedure.

3) the field computation inside the TLEC is performed with FIT [5];

4) the values of the $E$-field are exported over a regular grid;

5) the indexes of merit are applied to decide which are the better TL configurations.

The third and fourth steps are computed by using CST Microwave Studio (CST-MWS). In the end of the process, a file is exported to our optimization environment.

\section{B. Uniformity Index Calculation}

For the $x$-direction, the mean $E$-field $\left(\bar{E}_{x}\right)$, the standard deviation $\left(\sigma_{x}\right)$, and the standard deviation in decibels $\left(\hat{\sigma}_{x}\right)$ are calculated by

$$
\begin{aligned}
\bar{E}_{x} & =\frac{1}{N} \sum_{k=1}^{N} E_{x k} \\
\sigma_{x} & =\sqrt{\frac{1}{N-1} \sum_{k=1}^{N}\left(E_{x k}-\bar{E}_{x}\right)^{2}} \\
\hat{\sigma}_{x} & =20 \log _{10}\left(\frac{\bar{E}_{x}+\sigma_{x}}{\bar{E}_{x}}\right) .
\end{aligned}
$$

where $N$ is the number of $E$-field values inside the evaluated volume.

Similar equations could be obtained to the $y$-axis, $z$-axis and to the combined $E$-field [1], [2] and a maximum value equal to $4 \mathrm{~dB}$ for the standard deviation is assumed in order to define the $\mathrm{WV}$ at frequencies lower than $80 \mathrm{MHz}$.

\section{Characterization Problem}

The characterization problem establishes how the TLEC works when associated to a set of transmission line positions and related WVs. We know that when the WV increases the mean electric field decreases. This means that a multiobjective optimization algorithm should be employed (two or more antagonistic goals), and this inner problem has six optimization variables defining the $\mathrm{WV}$ : two equatorial radii, polar radius $\left(r_{x}, r_{y}, r_{z}\right)$, and three parameters associated to the ellipsoid translation (shift) from the chamber's centre $\left(\Delta_{x}, \Delta_{y}, \Delta_{z}\right)$. In this set, some WV has high electric field, whereas the volume is low. There are other WVs, which belong to this set, but have low electric field and high volume. Inside the WVs the field homogeneity is constrained by predefined values $(4 \mathrm{~dB})$. When the chamber characterization is performed, the engineer could make a choice to obtain the most suitable set of TL to design

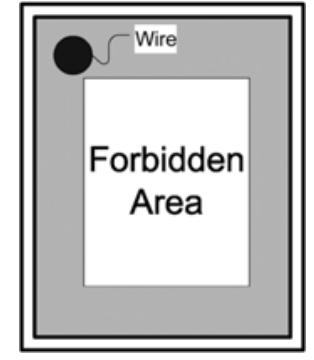

Fig. 3. Chamber cross section: the wire position.

the TLEC. In order to solve it, the characterization problem is written as an unconstrained optimization problem, where inequality constraint was treated as a penalty function. The conductor position problem could be written as an optimization problem as follows:

$$
\begin{gathered}
\left\{\begin{array}{l}
\operatorname{Max} \text { working volume } \\
\operatorname{Max} \bar{E}_{x, y, z}
\end{array}\right. \\
\text { submitted to: } \operatorname{Max}\left(\hat{\sigma}_{x}, \hat{\sigma}_{y}, \hat{\sigma}_{z}, \hat{\sigma}_{x, y, z}\right) \leq 4 \mathrm{~dB} .
\end{gathered}
$$

In order to bound the optimization parameters, Fig. 3 shows a cross section of the chamber that should be analyzed. First, each wire is parallel to one of the three axes of the chamber as Fig. 1 shows. So, each wire could only be placed in the gray region, because it could not be located close to the wall, due to the standards, and if we put it in the center of the chamber (the forbidden area) the associated WV will be small. It should be considered as well that the TLs cannot be crossed. So every TL can be moved only inside a space near the walls to avoid solutions that are impossible for practical implementation.

The solution of the optimization problem stated by (4) is then obtained by the calculation of fields and standards deviations in $x$-direction, as proposed in (1)-(3). The fields and standard deviations in other directions must also be calculated and have similar expressions.

Multiobjective optimization (4) seeks to characterize the components of a vector-valued cost function. Unlike single objective optimization, the solution to this problem is not a single point, but a family of efficient points called Pareto front. Each point on this surface is optimal because no improvement can be achieved in a cost vector component that does not lead to degradation in at least one of the remaining components. Each element in the efficient set constitutes a nondominated (noninferior or nonsuperior) solution to the multiobjective problem. With this set of solutions it is possible to understand the dependence between each objective. The Pareto set of this multiobjective problem was obtained by using the multiobjective genetic algorithm (MGA) [4]. The MGA is derived from the genetic algorithm (GA), which is a stochastic procedure based on the concepts of natural selection in genetics.

The EMC standards adopt a parallelepiped as a WV [7]. In this work, we have adopted another solid to characterize the volume: an ellipsoid [2].

\section{Index of Merit (LA)}

The index of merit LA is able to perform TLEC configuration comparison in its flexibility. This index consists of the area 


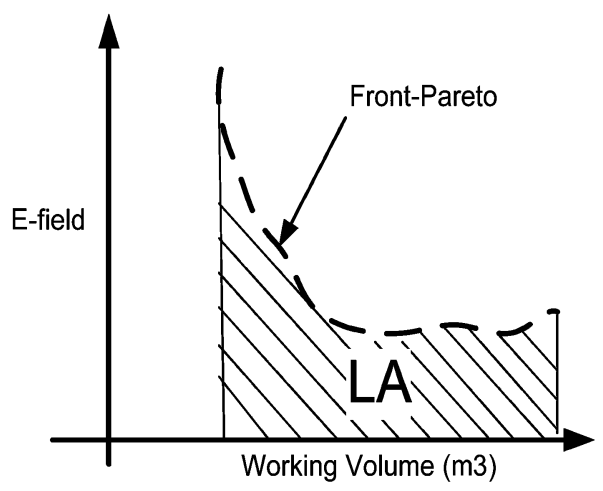

Fig. 4. Index of merit LA.

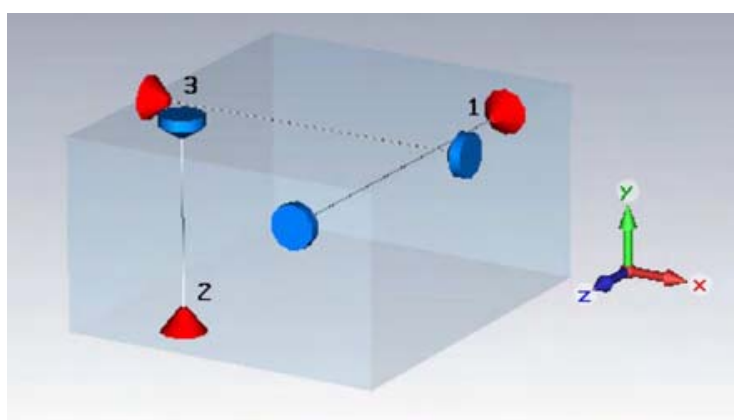

Fig. 5. Transmission lines positioning for characterization process.

under the Pareto front, as shown in Fig. 4, calculated for each individual of optimization problem (TL configuration). Then, this area can be used as a sensitive index, which averages the WV and the $E$-field within the chamber.

\section{RESULTS}

\section{A. Characterization Problem}

The characterization problem was solved applying MGA [2], [4] considering 500 individuals with ellipsoid characteristics $\left[r_{x}, r_{y}, r_{z}, \Delta_{x}, \Delta_{y}, \Delta_{z}\right]$, over 20 generations; crossover $=$ $50 \%$; mutation $=80 \%$; applying elitism has the following variables limits minimum: $[0,0,0,-2.6,-2.3,-1.35]$ and maximum: [2.6, 2.3, 1.35, 2.6, 2.3, 1.35].

For the sake of an example, we can take the characterization results concerning an individual with TLs positions $x_{1}=3.5 \mathrm{~m}$, $y_{1}=2.8 \mathrm{~m}, x_{2}=1.04 \mathrm{~m}, z_{2}=4.03 \mathrm{~m}, y_{3}=2.56 \mathrm{~m}$, and $z_{3}=$ $2.91 \mathrm{~m}$, as shown in Fig. 5.

After the $E$-field calculation using CST-MWS for this specific individual, we retrieve the $E$-field values at each point in TLEC and start the characterization process. The MGA algorithm is applied to search the mean $E$-field and WVs that are the dominant ones for this TLEC configuration. The resulting dominant WVs are show in Fig. 6, and the related Pareto front is shown in Fig. 7.

\section{B. Optimization Problem}

Once we can characterize each TLEC configuration, we perform the optimization process for TLEC with its dimensions $(5.20 \mathrm{~m} \times 4.55 \mathrm{~m} \times 2.70 \mathrm{~m})$ by applying the mono-objective GA, considering 15 individuals with TL characteristics $\left(x_{1}, y_{1}\right.$, $x_{2}, z_{2}$, and $\left.y_{3}, z_{3}\right)$.

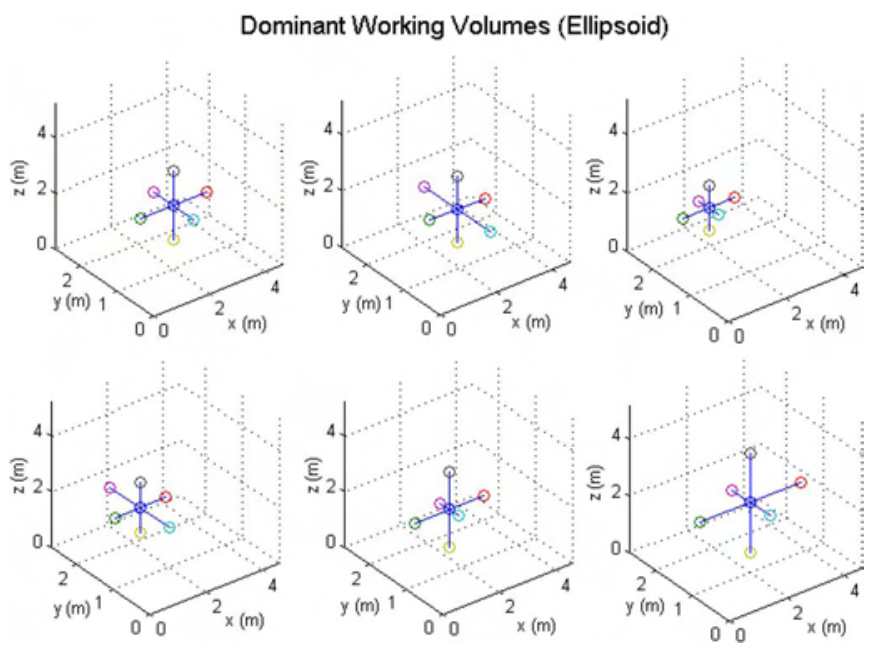

Fig. 6. Resulting dominant WV for characterization process.

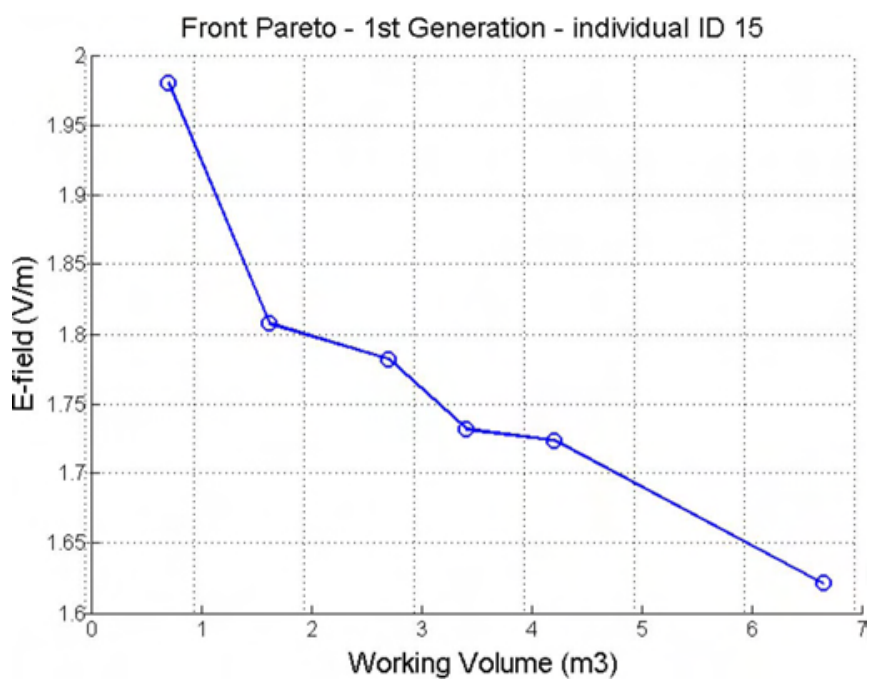

Fig. 7. Resulting Pareto front for characterization process.

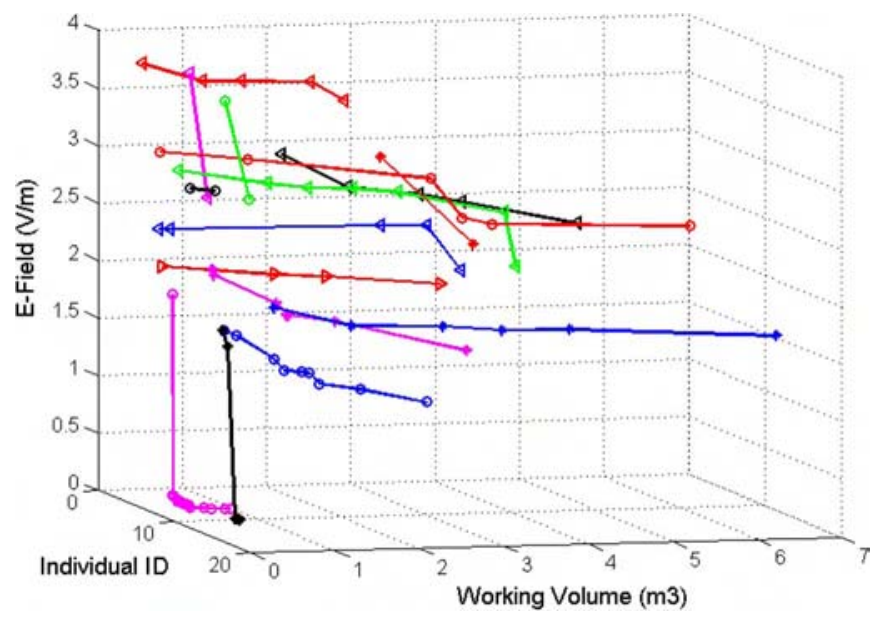

Fig. 8. Resulting Pareto front for the first generation from optimization process.

For the first generation (15 individual TLEC configurations), we have 15 Pareto front (see Fig. 8, which shows the Pareto sets according to its ID number). This results in the LA index values (Fig. 9) for the optimization process. One can realize that for each Pareto front the LA index has different values, because the area under the Pareto front, when the $E$-field and WV are considered, has a strong variation. 


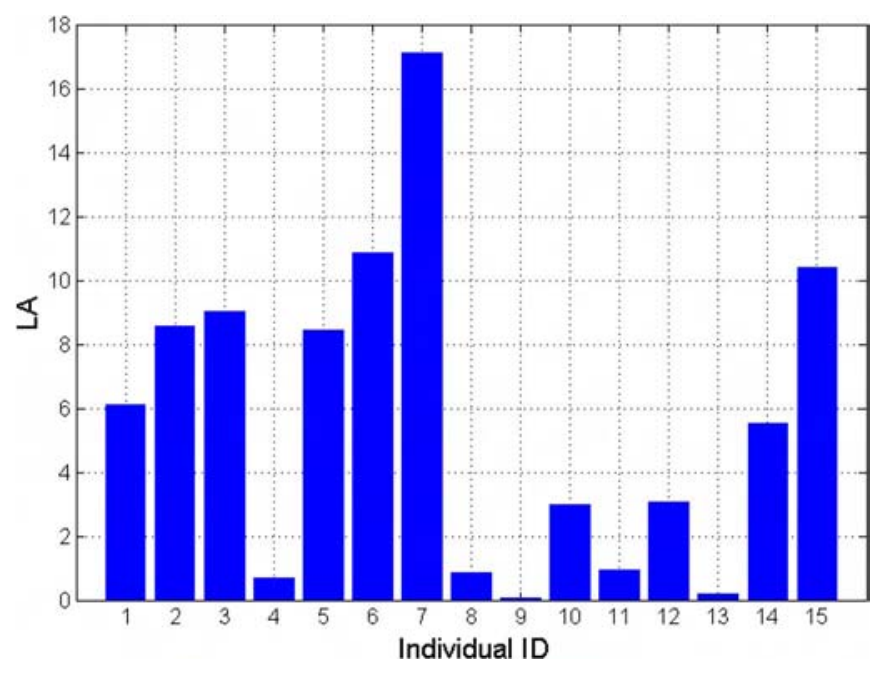

Fig. 9. LA indexes of merit calculated for each TLEC configuration of optimization process first generation.

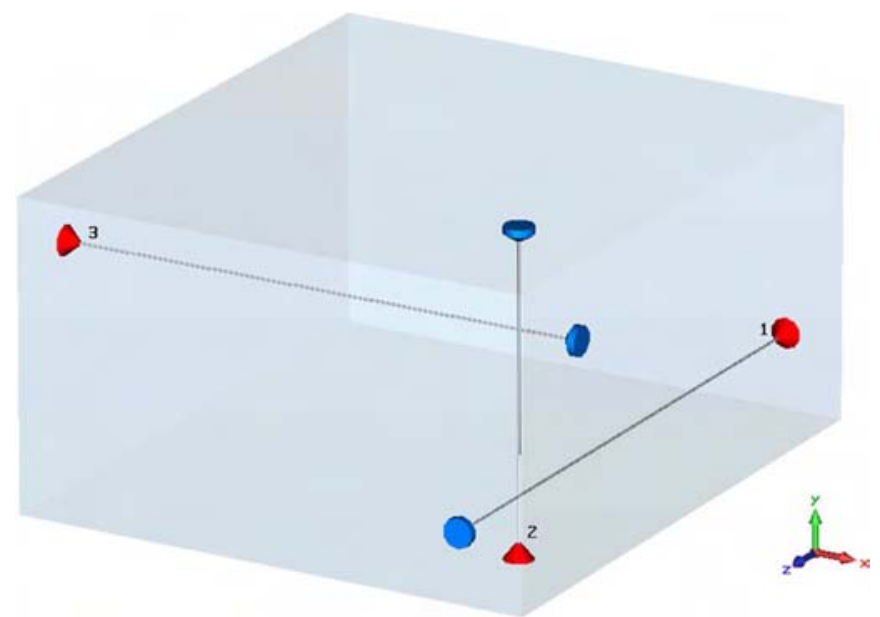

Fig. 10. Best individual calculated by hybrid method.

After ten generations of optimization process, we have made the evaluation/characterization of 150 individuals, and the final index of merit for the best individual LA $=27.1$ and the TLs positions are $x_{1}=3.72 \mathrm{~m}, y_{2}=2.46 \mathrm{~m}, x_{2}=0.62 \mathrm{~m}, z_{2}=$ $4.12 \mathrm{~m}, y_{3}=0.62 \mathrm{~m}$, and $z_{3}=4.23 \mathrm{~m}$ (Fig. 10).
The hybrid method advantages are to allow a strong flexibility when choosing the TLs configurations, as well as we have several TLs configurations that present a fitness a little bit lower than the best one (LA with values among 20 and 27), but that reach better the user desire and necessity. Then, the TLEC user and the TLEC designer can choose among the solutions the one that has a greater mean $E$-field and low $\mathrm{WV}$, or a WV that has a lower $E$-field but greater WV.

When we remember that the best individual of the first generation has LA value near 17 for the seventh individual (Fig. 9), we have an improvement of $63 \%$ at LA index in the end of the optimization process.

\section{CONCLUSION}

A mono-objective GA is performed as an outer layer in order to optimize the TL positions. As inner layers, first we perform the $E$-field calculation by using CST-MWS, and after, the chamber characterization by applying oriented search method (MGA) which results in a Pareto front to each evaluated configuration. In order to compare the different TL configurations, the area under the Pareto front was proposed as the index of merit (LA). To sum up, the proposed hybrid method was used to optimize the chamber configuration, by using the criteria (LA) to compare TL configurations (individual).

\section{REFERENCES}

[1] D. Weinzierl et al., "Numerical evaluation of non-canonical reverberation chamber configurations," IEEE Trans. Magn., vol. 44, no. 6, pp. 1458-1461, 2008.

[2] S. L. Avila et al., "Maximum working volume evaluation in a noncanonical reverberation chamber," IEEE Trans. Magn., vol. 45, no. 3, pp. 1646-1649, 2009.

[3] J. Perini and L. S. Cohen, "Extending the frequency of mode stir chambers to low frequencies," in Proc. IEEE Int. Symp. Electromagn. Compat., 2000, vol. 2, pp. 633-637.

[4] S. L. Avila et al., "Sensitivity analysis in the parameters space applied to decision making in multi-objective evolutionary optimization," IEEE Trans. Magn., vol. 42, no. 4, pp. 1103-1106, 2006.

[5] CST Microwave Studio, "Advanced topics," [Online]. Available: www. cst.com

[6] T. Weiland, "Time domain electromagnetic field computation with finite difference methods," Int. J. Num. Mod., ENDF, vol. 9, pp. 259-319, 1996.

[7] Electromagnetic Compatibility (EMC), Part 4, Testing and Measurement Techniques-Section 21: Reverberation Chamber Test Methods, IEC61.000-4-21. 